\title{
Short-term Load Forecasting for Microgrids Based on Discrete Wavelet Transform and BP Neural Network
}

\author{
Chong Gao*, Yuan-sheng Huang* and Hai-feng Wang*
}

Department of Economic Management,North China Electric Power University, Baoding, 071003, P. R. China

\begin{abstract}
Electricity is of great vital and indispensable to national economies. A new short-term load forecasting for micro grid is proposed in this paper. After comparing and analyzing all load characteristic in the time domain and frequency domain, we apply wavelet transform to decompose the load signal. After that, the training set and text set are selected in consideration of the effects generated by the temperature and day type. At length, BP natural network is employed you forecast the micro grid load. The final result proves that the forecasting precision of the method we propose is obviously better than the traditional ones. What's more, our method has Strong adaptability and good generalization ability.
\end{abstract}

Keywords: Micro-grid, load forecasting, wavelet transform, BP netural network.

\section{INTRODUCTION}

As we all know, electricity is indispensable and of strategic importance to national economies [1]. However, low carbon is required in modern society, that is why national administrations and international institutions pay more attention to development of microgrid. Some Demonstration Project are the Kyoto, Japan [2], the Labein, Spanish [3], or Mad River, America [4]. A microgrid consists of a set of dispatchable (turbines, reciprocating engines, fuel cells) and/or non-dispatchable generators (wind turbines, PV), electrical and thermal energy storage, a grid connection for import and/or export of electricity, heat and power distribution infrastructure and an energy management system [5]. A example of microgrid including DI, Distributed Generation (DG), end-point and storage elements is showed in Fig. (1).

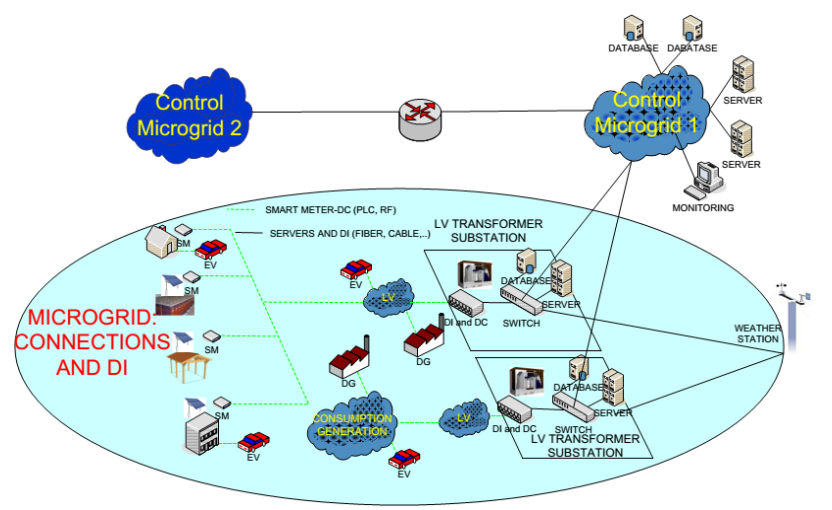

Fig. (1). Microgrid example.

\section{WAVELET TRANSFORM}

Wavelet transform can divide a function or signal into different frequency components, and then study the various parts of it. The most commonly used set of discrete wavelet transforms was formulated by Ingrid Daubechies in 1988 [6]. The filter bank implementation of wavelets can be interpreted as computing the wavelet coefficients of a discrete set of child wavelets for a given mother wavelet $\psi(t)$.In the case of the discrete wavelet transform, the mother wavelet is shifted and scaled by powers of two

$\psi_{j, k}(t)=\frac{1}{\sqrt{2 j}} \psi\left(\frac{t-k 2^{j}}{2^{j}}\right)$

Where $j$ is the scale parameter and $k$ is the shift parameter, both which are integers.

Recall that the wavelet coefficient $\gamma_{\text {of a signal }} x(t)$ is the projection of $x(t)$ onto a wavelet, and let $x(t)$ be a signal of length $2^{N}$. In the case of a child wavelet in the discrete family above,

$\gamma_{j k}=\int_{-\infty}^{\infty} x(t) \frac{1}{\sqrt{2 j}} \psi\left(\frac{t-k 2^{j}}{2^{j}}\right) d t$

Now fix $j$ at a particular scale, so that $\gamma_{j k}$ is a function of $k$ only. In light of the above equation, $\gamma_{j k}$ can be viewed as a convolution of $x(t)$ with a dilated, reflected, and normalized version of the mother wavelet, $h(t)=\frac{1}{\sqrt{2 j}} \psi\left(\frac{-t}{2^{j}}\right)$ sampled at the points $1,2^{j}, 2^{2 j}, \ldots, 2^{N}$. But this is precisely what the detail coefficients give at level 
$j$ of the discrete wavelet transform. Therefore, for an appropriate choice of $h[n]$ an $g[n]$, for a given mother wavelet $\psi(t)$ the detail coefficients of the filter bank correspond exactly to a wavelet coefficient of a discrete set of child wavelets [7].

\section{BP NEURAL NETWORK}

Artificial computational neural networks (CNNs) can work as analogous mechanisms of the biological nervous systems [8]. BP neural network is also called Error Back Propagation Neural Network. It is the most widely used in multilayer feedforward neural networks, which generally includes input layer, hidden layer and output layer.

Each basic BP neuron usually has $n$ input $X=\left(x_{1}, x_{2}, \ldots, x_{n}\right)$, and each input connects to the next layer through an appropriate weight $W=\left(w_{1}, w_{2}, \ldots, w_{n}\right)$ and threshold $b$. A example of basic BP neuron is shown in Fig. (2).

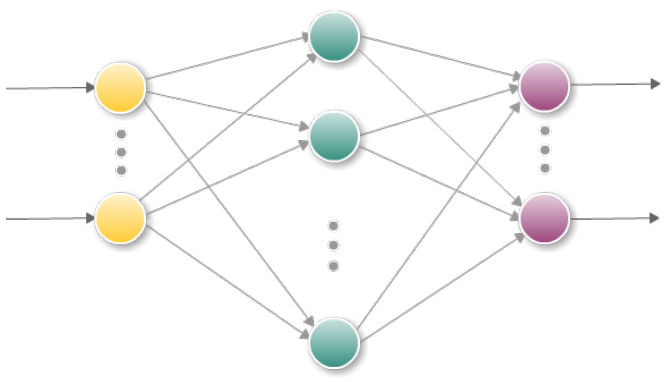

Input layer Hidden layer Output layer

Fig. (2). BP neuron.

The output function network is (3). What's more, the function belongs to monotone increasing function.

$y=f\left(\sum_{i}^{n} w_{1 i} x_{i}+b\right)$

\section{THE LOAD CHARACTERISTIC ANALYSIS OF MICROGRID}

Short-term load forecasting is the premise that power system can operate economically and reliably. The existing forecasting methods mainly are time series method, neural network method and Auto-Regressive and Moving Average Model (ARMA). Electric load has a special periodic load which can waves in days or weeks or year in cycle and small cycles make up big one. A mixed-signal consists of different frequency signals. The wavelet transform can decompose the mixed-signal into block signals of different frequency bands. So applying wavelet transform to load sequence can projected the load sequence to different scales, respectively. Such sequences on each scale can represent the original sequence of different "frequency domain" component. In this way, they demonstrate more clearly the cyclical of load sequence.

\section{MODELING}

\subsection{The Model of Wavelet Transform}

Compared with the traditional power grid, microgrid is concerned as variable load connected to the large grid and the fluctuation range of microgrid is large, especially in the high frequency region. In this paper, the volatility of load characteristics in microgrid and traditional power grid are compared in two ways by the time domain and frequency domain with the aid of wavelet transform. Applying wavelet transform to the power load. That is to say, the load is about to carry out a multi-layer decomposition. And it can show the trends of the load signal with various cycles clearly and predict it.

When analysing the time domain, the load characteristic curve are considered as time series, and after normalized it can calculate the standard deviation $\alpha$ of each loading curve in standard sequence time series grads in the time domain[9]. The main analysis is the high-frequency component of the time series when analyzing the frequency. The mutagenicity of high frequency component of the signal is strong in the time domain. For the power load time series we selected, we can employ discrete wavelet transform to conduct spectral analysis. Here, we decompose the power load time series into three layers. The result is displayed in Fig. (3). In Fig. (3), $A$ represents the low-frequency component and $D$ represents the high-frequency component of corresponding layer. The relationship among them is $S=A_{3}+D_{1}+D_{2}+D_{3}$. We define $\beta$ as the ratio of energy of high-frequency component in the first layer to the energy of the whole signal. Microgrid load is less stable during peak and trough, and the mutagenicity of high frequency component is strong.

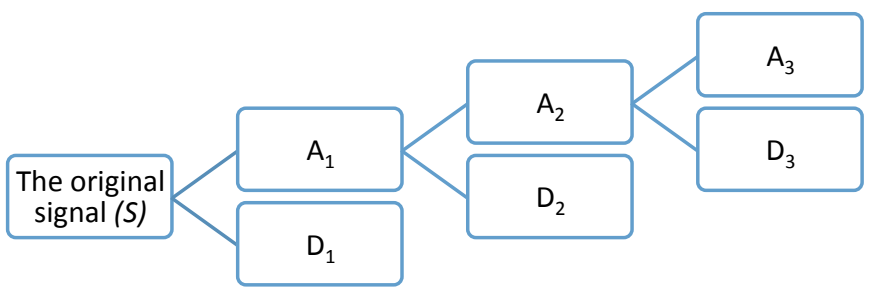

Fig. (3). The decomposition of power load.

\subsection{The Model of BP Neural Network}

For each neural network forecasting model, we choose different training samples according to which categories [10]. We divide the data of each layer into training set and test set. We assume the training set of component in each layer as follows:

(1) Among the trainging set, the load data of decomposition components in the ith hour, the kth layer, the nth training day is 
$P=\left\{P_{k, 1,1}, P_{k, 1,2}, P_{k, 1,3}, \ldots, P_{k, 1,24}, P_{k, 2,1}, P_{k, 2,2}, \ldots, P_{k, 2,24}, \ldots, P_{k, n, 1}, P_{k, n, 2}, \ldots, P_{k, n, 24}\right\}$

$k=1,2,3,4$

(2) The data of temperature in the nth training day is $B=\left\{B_{1, j}, B_{2, j}, \ldots, B_{n, j}\right\}, j=1,2,3$

Where $B_{n, 1}$ stands for the highest temperature in the nth training day, $B_{n, 2}$ stands for the lowest temperature in the nth training day, $B_{n, 3}$ stands for the average temperature in the nth training day.

(3) $W=\{1,2,3,4,5,6,7\}$ stand for the day type of training days and predicting days respectively.

(4) $T=\left\{T_{1}, T_{2}, T_{3}\right\}$ is the data of temperature in the predicting days. $T_{1}$ is highest temperature, $T_{2}$ is the lowest and $T_{3}$ is the average.

If every day were seen as a different type in a week, then there is a total of seven types. And every hour of electricity load is measured once a day, which is the predicting day before, so there is a total of 24 sets of load data. Assume the mutation doesn't occur between adjacent points load values, then the later time value and the previous value is relevant, so we put the load data in real time from the previous day as samples of network data.

Besides, due to the load associated with temperature, we put the day atmosphere characteristic of power load forecasting data, which are gotten from weather forecasts predict including daily maximum and minimum temperatures, as input variables of a network. To sum up, the input variables is a 26-dimensional vector. As for output variables, what we need is day electric load forecasting, so the output variable is several 24-dimensional vectors in four levels.

\section{NUMERICAL CALCULATION}

In this paper, microgrid demonstration project is selected in a particular city May 2013, and two weeks before the load data are selected as training sets, a day after $24 \mathrm{~h}$ loads data as the test set. Initial training set image is shown in Fig. (4) and each layer component curve with Wavelet Decomposition can be seen in Fig. (5). Meanwhile, we get minimum and maximum temperatures for training day and testing day from China Meteorological Administration.

When establishing the BP network model, we need to obtain the input and output variables normalized, to transfer data to the interval $[0,1]$. In the paper, we use the function as follows:

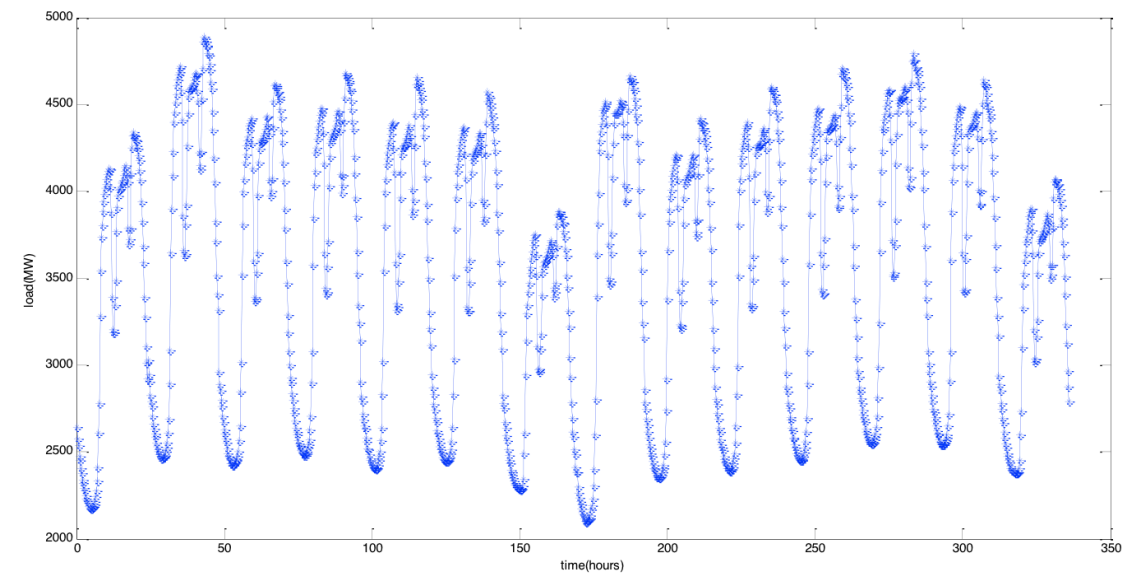

Fig. (4). Microgrid daily load curve.
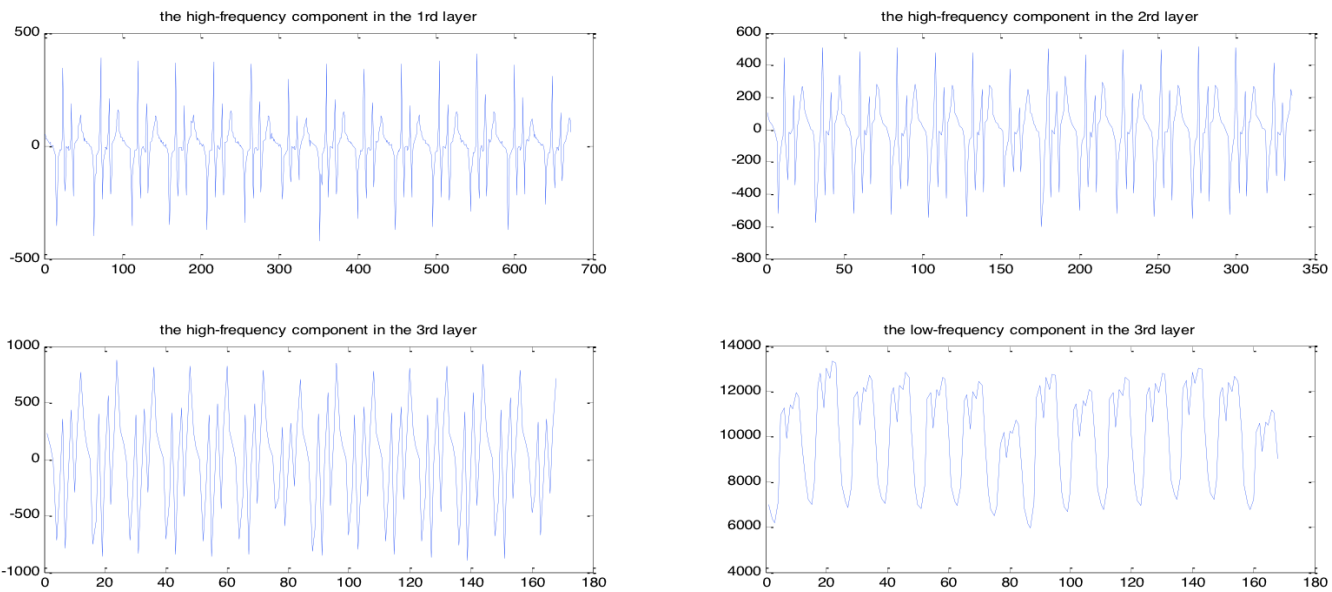

Fig. (5). The decomposed components of microgrid load data. 
$\hat{x}=\frac{x-x_{\min }}{x_{\max }-x_{\min }}$

In addition, the network should take a lot of training before they can be used for the practical application of the power load forecasting. Considering the structure of the network is more complex, a lot number of neurons are needed, proper training times and learning rate are requred. Table $\mathbf{1}$ shows the training parameters.

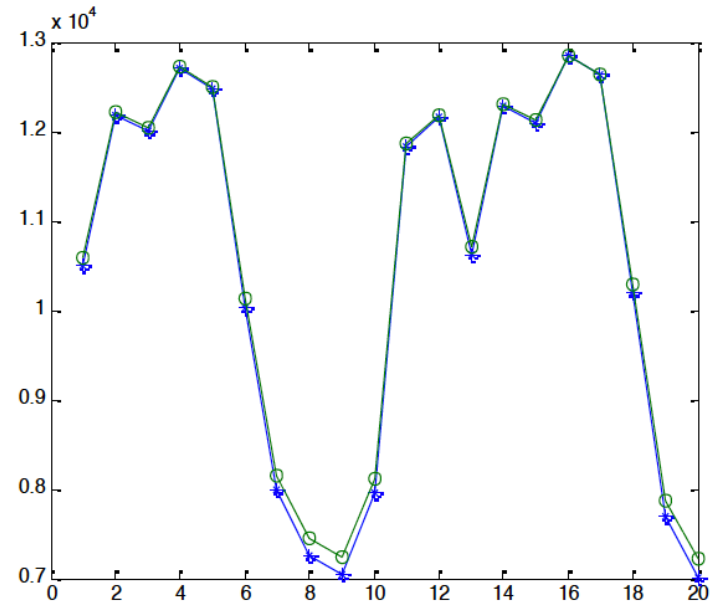

The prediction of sequence a 3

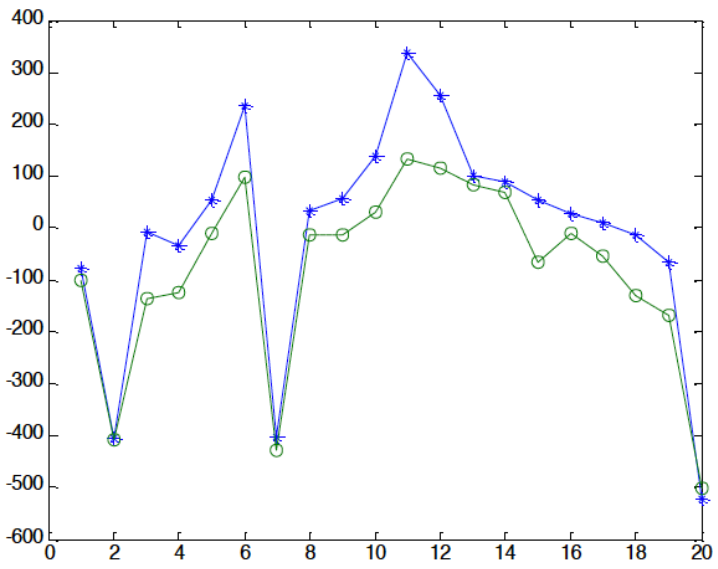

The prediction of sequence $\mathrm{d} 2$

Fig. (6). The prediction results.

\section{CONCLUSION}

A new short-term load forecasting for microgrids is proposed in this paper. After comparing and analyzing all load characteristic in the time domain and frequency domain. We apply wavelet transform to decompose the load signal. After that, the traning set and text set are seclected in consideration of the effects generated by the temperature and day type. At length, BP netural network is employed yo forcast the microgrid load. The final result proves that the forecasting pre-
Table 1. Training parameters.

\begin{tabular}{|c|c|c|}
\hline Training times & Training goals & Learning Rate \\
\hline \hline 1000 & 0.01 & 0.1 \\
\hline
\end{tabular}

Using SIM simulation functions to calculate the output of the network, we get the results as shown in Fig. (6). Diagram shows the relative error of this method each forecast point are within the $2 \%$, showing that predictions are very accurate.

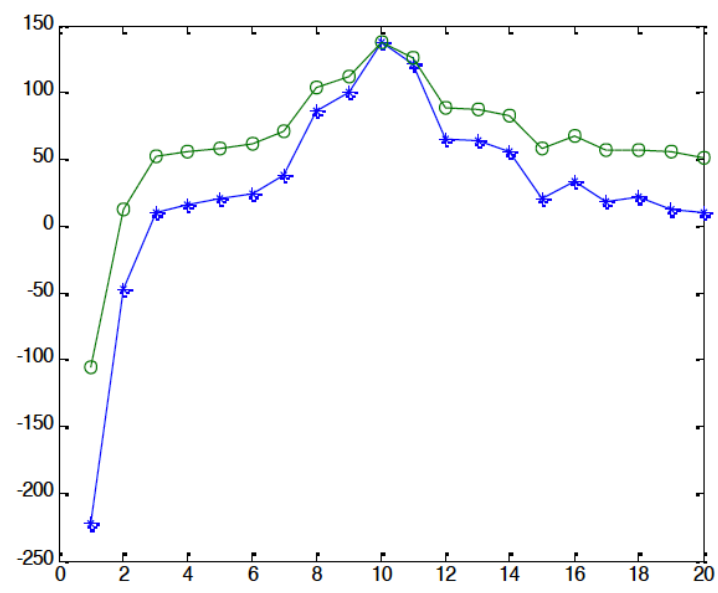

The prediction of sequence $\mathrm{d} 1$

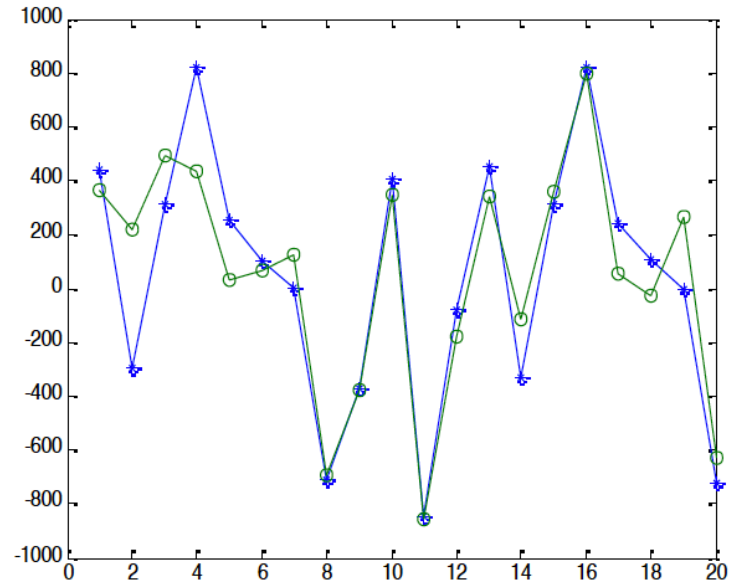

The prediction of sequence $\mathrm{d} 3$

cision of the method we propose is obviously better than the traditional ones. What's more, our method has strong adaptability and good generalization ability.

\section{CONFLICT OF INTEREST}

The authors confirm that this article content has no conflicts of interest. 


\section{ACKNOWLEDGEMENTS}

This study is supported by "the Fundamental Research Funds for the Central Universities (No.13MS112)", "the National Natural Science Foundation of China (NSFC) (No.71471061)" and "Soft Science Research Base of Hebei Province".

\section{REFERENCES}

[1] Hernandez L., Baladrón C., Aguiar J. M., Carro B., SanchezEsguevillas A. J., and Lloret J. "Short-term load forecasting for microgrids based on artificial neural networks", Energies, vol. 6, no. 3, pp. 1385-1408, 2013.

[2] Funabashi T. and Yokoyama R. Microgrid field test experiences in Japan. In Power Engineering Society General Meeting, 2006. IEEE (pp. 2-pp). IEEE, June 2006.

[3] Bernaras A., Laresgoiti I., Bartolome N., and Corera J. "An ontology for fault diagnosis in electrical networks". In Intelligent Systems Applications to Power Systems, 1996. Proceedings, ISAP'96., International Conference on (pp. 199-203). IEEE, February 1996.
[4] Su W. and Wang J. "Energy management systems in microgrid operations", The Electricity Journal, vol. 25, no. 8, pp. 45-60, 2012.

[5] Hawkes A. D. and Leach M. A. "Modelling high level system design and unit commitment for a microgrid", Applied energy, vol. 86, no. 7, pp. 1253-1265, 2009.

[6] Daubechies

wavelet.https://en.wikipedia.org/wiki/Daubechies wavelet, note="[Online; accessed 14-March-2015]".

[7] Discrete wavelet transform. https://en.wikipedia.org/wiki/ Discrete_wavelet_transform\#Daubechies_wavelets, te $=$ "[Online; accessed 14-March-2015]".

[8] Mikko Kivela, Alex Arenas, Marc Barthelemy, James P Gleeson, Yamir Moreno, and Mason A Porter. "Multilayer networks", Journal of Complex Networks, vol. 2, no. 3, pp. 203-271, 2014.

[9] Garcia M. P. and Kirschen D. S. "Forecasting system imbalance volumes in competitive electricity markets"[C]/Power Systems Conference and Exposition, 2004. IEEE PES. IEEE: pp. 1805$1812,2004$.

[10] Y. W Chen and L. Y. Wang. "Short-term load forecasting model based on Combined neural network", China Electrical Engineering, vol. 21 , no. 4 , pp. 79-82. 2001

(C) Na et al.; Licensee Bentham Open.

This is an open access article licensed under the terms of the Creative Commons Attribution Non-Commercial License (http://creativecommons.org/licenses/by$\mathrm{nc} / 3.0 /$ ) which permits unrestricted, non-commercial use, distribution and reproduction in any medium, provided the work is properly cited. 